\title{
Coherent Acceptability Measures in Multiperiod Models
}

\author{
Berend Roorda* Hans Schumacher ${ }^{\dagger} \quad$ Jacob Engwerda ${ }^{\ddagger}$
}

Final revised version, September $2004^{\S}$

\begin{abstract}
The framework of coherent risk measures has been introduced by Artzner et al. (1999) in a single-period setting. Here we investigate a similar framework in a multiperiod context. We add an axiom of dynamic consistency to the standard coherence axioms, and obtain a representation theorem in terms of collections of multiperiod probability measures that satisfy a certain product property. This theorem is similar to results obtained by Epstein and Schneider (2003) and Wang (2003) in a different axiomatic framework. We then apply our representation result to the pricing of derivatives in incomplete markets, extending results by Carr et al. (2001) to the multiperiod case. We present recursive formulas for the computation of price bounds and corresponding optimal hedges. When no shortselling constraints are present, we obtain a recursive formula for price bounds in terms of martingale measures.
\end{abstract}

Keywords: coherent risk measures; acceptability measures; dynamic consistency; robustness; option pricing; incomplete markets.

JEL Classification: G11; G13.

*B. Roorda, FELab and Department of Finance and Accounting, University of Twente, P.O. Box 217, 7500 AE, Enschede, the Netherlands. Phone: +3153-4894383. E-mail: b.roorda@utwente.nl. This research was supported by the Netherlands Organization for Scientific Research (NWO - MaGW/ESR, project number 510-01-0025).

${ }^{\dagger}$ J.M. Schumacher, Department of Econometrics and Operations Research, Tilburg University, P.O. Box 90153, 5000 LE Tilburg, the Netherlands.

${ }^{\ddagger}$ J.C. Engwerda, Department of Econometrics and Operations Research, Tilburg University, P.O. Box 90153, 5000 LE Tilburg, the Netherlands.

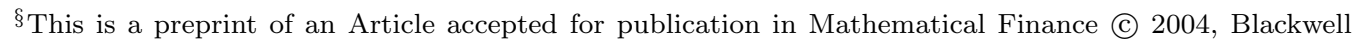
Publishing, Inc. 


\section{Introduction}

The seminal work of Artzner et al. (1999) on coherent risk measures is focused primarily on supervisory applications. Specific examples discussed in the paper concern the rules for capital adequacy in the 1996 Amendment to the 1988 Basel Accord, and the margin rules of the Chicago Mercantile Exchange and the US Securities and Exchange Commission. The axioms for acceptability that were put forward by Artzner et al. differ from the ones that have been used traditionally in statistical decision theory. Yet it is clear that the regulatory issues can be formulated as decision problems, and moreover there are many other situations where both the classical axioms of decision theory and the coherence axioms adopted by Artzner et al. can be taken into consideration. One may for instance think of capital budgeting decisions, the determination of premia for insurance contracts, and the pricing of derivatives in incomplete markets.

The coherence axioms in Artzner et al. (1999) lead to acceptability measures of the form

$$
\phi(X)=\inf _{\mathbb{P} \in \mathcal{P}} E_{\mathbb{P}} X
$$

where $X$ is a function from a (finite) set $\Omega$ to $\mathbb{R}$, and $\mathcal{P}$ is a class of probability measures on $\Omega .{ }^{1}$ One may compare (1.1) to the criterion obtained by Gilboa and Schmeidler (1989) from a set of decision-theoretic axioms. The Gilboa-Schmeidler criterion corresponds to a utility specification of the form

$$
U(X)=\inf _{\mathbb{P} \in \mathcal{P}} E_{\mathbb{P}}[u(X)]
$$

where $X$ and $\mathcal{P}$ are defined as above, and where $u(\cdot)$ is a utility function. The interpretation given by Gilboa and Schmeidler to the class of probability measures $\mathcal{P}$, and which their axiom system is designed to reflect, is that this class represents ambiguity in the sense of Ellsberg (1961). There is no explicit appeal to ambiguity in Artzner et al. (1999). The suggested interpretation of the class $\mathcal{P}$ in this paper is just that it represents a set of (generalized) scenarios to be used in risk assessment.

Depending on one's perspective, the Gilboa-Schmeidler robust expected utility framework might be described as "richer" or as "more complicated" than the coherence framework of Artzner et al. (1999). Of course, in view of the many different situations to which statistical decision theory applies, it would be pointless to argue a preference for either of the

\footnotetext{
${ }^{1}$ Actually the paper Artzner et al. (1999) uses risk measures rather than acceptability measures. We changed the terminology here to facilitate comparison with common formulations in decision theory, and also to stress the applicability of the same notion in different contexts. The difference between the risk measures $\rho(X)$ of Artzner et al. (1999) and the acceptability measures $\phi(X)$ used here is just a change of sign $(\phi(X)=-\rho(X))$. For simplicity reasons we ignore discounting. Artzner et al. (2003) use the term "risk adjusted values" for what we call acceptability measures.
} 
two frameworks over the whole range of applications; we come back below to a discussion of specific circumstances in which the coherence axioms may be reasonable. From a purely technical point of view, the coherence framework offers substantial simplifications with respect to the robust expected utility framework. In fact, the decision problem in Gilboa and Schmeidler (1989) is formulated in terms of the randomization device that was introduced by Anscombe and Aumann (1963) to simplify Savage's (1954) axiomatization of subjective expected utility. Casadesus-Masanell et al. (2000) succeeded in obtaining a derivation of the Gilboa-Schmeidler utility specification without making use of Anscombe-Aumann lotteries. Compared to the long proofs in Gilboa and Schmeidler (1989) and Casadesus-Masanell et al. (2000), the coherence criterion (1.1) can be obtained in a relatively straightforward way from standard results on the representation of convex sets. As noted in Artzner et al. (1999), this was in fact already done by Huber (1981) in the context of robust statistics.

Several proposals have been made in recent years that aim at expressing ambiguity aversion in a continuous-time environment. A "robust control" framework that penalizes deviations from a reference measure has been developed for this purpose by Hansen, Sargent, and co-authors; see for instance Hansen and Sargent (2001), Hansen et al. (2001). Chen and Epstein (2002) have formulated a continuous-time multiple-priors utility specification, following the recursive multiple-prior specification for discrete-time decision problems of Epstein and Wang (1994). The Hansen-Sargent framework has been adapted for applications in portfolio choice by Maenhout (2004), and this adaptation was extended further by Uppal and Wang (2003). While these adaptations certainly add to the tractability of the resulting optimization problems, they lead to difficulties in interpretation and it has been questioned by Pathak (2002) whether the original robustness motivation is still valid under Maenhout's transformation. The robust control framework can be linked to the stochastic differential utility of Duffie and Epstein (1992); see Maenhout (2004), Hansen et al. (2001), and Skiadas (2003). None of the various proposals for continuous-time versions of the Gilboa-Schmeidler specification has been supplied with an axiomatic foundation. However, axiom systems that support discrete-time recursive multiple-priors utility have been proposed recently by Wang (2003) and by Epstein and Schneider (2003).

In this paper we consider an axiom scheme that supports an extension of coherent acceptability measures to a multiperiod setting. As in Wang (2003) and Epstein and Schneider (2003), we limit ourselves to a finite-state setting (tree models). To the axioms of Artzner et al. (1999), we add an axiom of dynamic consistency which is similar to, although not quite the same as, the axioms of the same name that are used by Wang and by Epstein and Schneider. Roughly the axiom says that, if a position is acceptable at the next time instant under all possible scenarios, then this position is also acceptable now. We obtain a representation theorem for multiperiod acceptability measures that extends the atemporal result 
in Artzner et al. (1999). Similar representation theorems have been obtained independently by Artzner et al. (2003) and by Riedel (2003).

As is not surprising, both the formulation of the axiom systems and the proof of the representation theorem are considerably simpler in the multiperiod coherence framework than in the multiperiod Gilboa-Schmeidler framework. One may of course ask whether there are important applications in which the axioms of coherent acceptability measures can be viewed as reasonably applicable. In addition to the regulatory applications considered in Artzner et al. (1999), we would like to mention here the problem of derivative pricing in incomplete markets. In particular, we focus attention on markets that are incomplete due to the presence of unhedgable risks rather than to other factors such as illiquidity, inaccurate information, transaction costs, constraints on trading, or indivisibility of assets. As argued by Carr et al. (2001) in an atemporal setting, coherent acceptability measures may be applied to obtain price bounds for derivative assets in such markets. In particular the positive homogeneity axiom, ${ }^{2}$ which is debatable in the case of individual decision-making, can be seen as justified in the incomplete-market context because risks can be split over many market participants. The pricing of derivative assets in incomplete markets is in fact our main background motivation in this paper.

In addition to the axiomatics and the representation theorem, we consider in this paper also some issues related to the use of coherent acceptability measures. As noted above, acceptability measures can be employed to obtain price bounds for derivatives in incomplete markets. This follows a line of reasoning which has been employed by several authors, who have argued that price bounds for derivative assets in incomplete markets obtained from strict arbitrage arguments ("superreplication") are often too wide to be practically useful, and that tighter limits ("good-deal bounds") can be obtained from the use of suitable measures of acceptability. Examples include Cochrane and Saá Requejo (2000), Bernardo and Ledoit (2000), Jaschke and Küchler (2001), Černý and Hodges (2002). An alternative approach to pricing in incomplete markets can be based on calibration of a family of riskneutral processes to option prices; see for instance Hull and White (1990), Heston (1993), Madan et al. (1998).

The "good-deal" approach to pricing in incomplete markets does not require by itself that the acceptability measure used should be coherent, and not all authors work with coherent measures; for instance Cochrane and Saá Requejo (2000) use the Sharpe ratio. However, coherent acceptability measures do have a special relevance in that they create a direct link between the "good-deal" approach and the method based on calibration. Indeed, it has been shown by Carr et al. (2001) that absence of strictly acceptable opportunities, where

\footnotetext{
${ }^{2}$ The axiom of positive homogeneity states that the degree of acceptability of a position is proportional to its size; see Section 2.1 below.
} 
acceptability is defined in terms of a given coherent measure, implies that the pricing measure must be a nontrivial convex combination of the test measures used in the specification of the given acceptability measure. In this paper, we show that, under the dynamic consistency axiom, this result carries over to the multiperiod context. In other words, calibration over martingale measures can be restricted to the convex combinations of the probability measures that are used to define the acceptability measure. Therefore, if for a given market one finds a specification of an acceptability measure that is successful in that the bounds that it produces are confirmed by the data, then the collection of probability measures that is used in this specification is also a good candidate to serve as the basis of a calibration exercise aiming at obtaining more precise price estimates.

A third issue to be discussed in this paper concerns tractability. A key aspect of multiperiod models is the possibility of dynamic hedging, and more generally it is of interest to compute portfolio strategies that are optimal with respect to a given acceptability measure. Papers that consider optimal portfolio strategies for multiple-priors utility specifications in continuous time include Cvitanić and Karatzas (1999), Uppal and Wang (2003), and Kirch (2003). In the multiperiod setting of this paper, we show that dynamic consistency implies the existence of a recursive algorithm for the computation of optimal strategies.

The dynamic consistency axiom leads to a property of the representing collection of probability measures which we call the "product property." ${ }^{3}$ This property essentially means that the collections of probability measures that are used as test measures for a given acceptability measure must be constructed as products (in a suitable sense) of collections of single-period measures. The property simplifies specification considerably.

An aspect that we do not consider in this paper is learning. Neglect of this aspect may be viewed as defendable for instance in a world that is nonstationary on a long timescale and in which agents have already learned essentially everything that can be learned given the limits of inference. Of course, in other situations it can be important to consider learning; see Wang (2003) for a discussion in the multiperiod Gilboa-Schmeidler framework.

Several recent papers including Cvitanicć and Karatzas (1999) and Frittelli and Scandolo (2004) have considered risk measures that are "dynamic" in the sense that they refer to the acceptability of streams of payments rather than single payoffs, but not in the sense that the risk measure itself is a process. Artzner et al. (2003) take both aspects into account. In this paper we consider only payoffs at the final time, so our acceptability measures are only dynamic in the sense that they are defined as processes. One may incorporate intermediate cashflows by assuming that these are invested in a riskless asset that pays out at the final time; in this way any payment that is received at a non-final node is added to the payoff

\footnotetext{
${ }^{3}$ Terminology differs across papers: Epstein and Schneider (2003) use the term "rectangular", Artzner et al. (2003) speak of "multiplicative stability", and Riedel (2003) just uses "consistency."
} 
at all corresponding final nodes. In general such an assumption is somewhat arbitrary and may not be justified. However, in many cases it may be reasonable to assume that there is freedom in portfolio rebalancings so that any investments in riskless assets can be undone. With this proviso, intermediate payments can be easily incorporated in the setting of the present paper.

The paper is organized as follows. In Section 2, we consider the axiomatics, and prove the representation theorem. We then turn to pricing in incomplete markets and to optimal hedging in Section 3. The validity of the dynamic consistency axiom is discussed in Section 4, and conclusions follow in Section 5. Some of the technical proofs have been collected in an Appendix.

Throughout the paper we take the unit of accounting to be a suitably chosen numéraire. In this way the complexity of the notation is somewhat reduced; in particular there is no explicit mention of interest rates.

\section{Axiomatics and the representation theorem}

\subsection{Single-period setting}

Here we briefly review the axiomatic setting for risk measures that was proposed by Artzner et al. (1999), with some (simple) modifications: acceptability measures are used instead of risk measures, and we do not consider discounting. Let $\Omega$ be a finite set, say with $n$ elements. The set of all functions from $\Omega$ to $\mathbb{R}$ will be denoted by $\mathcal{X}(\Omega)\left(\simeq \mathbb{R}^{n}\right)$. An element $X$ of $\mathcal{X}(\Omega)$ is thought of as a representation of the position that generates outcome $X(\omega)$ when the state $\omega \in \Omega$ arises. An acceptability measure defined on $\Omega$ is a mapping from $\mathcal{X}(\Omega)$ to $\mathbb{R}$. The number $\phi(X)$ that is associated to the position $X \in \mathcal{X}(\Omega)$ by an acceptability measure $\phi$ is interpreted as the "degree of acceptability" of the position $X$. An acceptability measure is said to be coherent if it satisfies the following four axioms, where $\mathbb{1}: \Omega \rightarrow \mathbb{R}$ is defined by $\mathbb{1}(\omega)=1$ for all $\omega:$

- translation property: $\phi(X+\eta \mathbb{1})=\phi(X)+\eta$ for all $\eta \in \mathbb{R}$

- superadditivity: $\phi\left(X_{1}+X_{2}\right) \geq \phi\left(X_{1}\right)+\phi\left(X_{2}\right)$

- positive homogeneity: $\phi(\lambda X)=\lambda \phi(X)$ for all $\lambda \geq 0$

- monotonicity: $X \geq Y$ implies $\phi(X) \geq \phi(Y)$.

Here we write $X \geq Y$ if $X(\omega) \geq Y(\omega)$ for all $\omega \in \Omega$. A general motivation of the above principles is provided by Artzner et al. (1999).

The following result is fundamental. 
Theorem 2.1 (Artzner et Al. (1999, Prop. 4.1); Huber (1981, Prop. 10.2.1)) An acceptability measure $\phi$ defined on a finite set $\Omega$ is coherent if and only if there exists a family $\mathcal{P}$ of probability measures on $\Omega$ such that, for all $X \in \mathcal{X}(\Omega)$,

$$
\phi(X)=\inf _{\mathbb{P} \in \mathcal{P}} E_{\mathbb{P}} X
$$

If an acceptability measure $\phi$ satisfies (2.1), it is said to be represented by the family $\mathcal{P}$ of probability measures, and the probability measures in the collection $\mathcal{P}$ are sometimes referred to as "test measures" for $\phi$. The above theorem can be generalized to the case of infinite sample spaces $\Omega$ if either the representation by probability measures is replaced by a representation in terms of finitely additive measures, or a continuity property is added to the coherence axioms (see Delbaen (2002), Föllmer and Schied (2002a-c)).

If $\phi$ is a coherent acceptability measure, then $\phi(\eta \mathbb{1})=\eta$ for all $\eta \in \mathbb{R}$; this follows from the axiom of positive homogeneity (which implies $\phi(0)=0$ ) and from the translation property. For convenience we introduce a separate term for this property.

Definition 2.2 An acceptability measure $\phi$ on a sample space $\Omega$ is said to be normalized if $\phi(\eta \mathbb{1})=\eta$ for all $\eta \in \mathbb{R}$.

In particular, if the set $\Omega$ consists of only one element $\omega$, then the only normalized acceptability measure is $\phi(X)=X(\omega)$. The case in which all uncertainty has been resolved will be used below in the multiperiod context as a starting point for backward recursions.

\section{$2.2 \quad$ Multiperiod setting}

We now pass to a multiperiod setting. To keep the context as simple as possible we still work with a finite sample space $\Omega$ (following Artzner et al. (1999) and Carr et al. (2001)), but we consider each sample now as a discrete-time trajectory. We begin with introducing some notation and terminology that will be needed below.

\subsubsection{Notation and conventions}

Let $T$ be a positive integer indicating the number of time periods over which we consider our economy. Let $A$ be a finite set which we shall refer to as the "event set." ${ }^{4}$ Define $\Omega$ as the set of all sequences $\left(\alpha_{1}, \ldots, \alpha_{T}\right)$ with $\alpha_{i} \in A$; we refer to such sequences as "full histories." The collection of sequences $\left(\alpha_{1}, \ldots, \alpha_{\tau}\right)$ of length $\tau(1 \leq \tau \leq T)$ will be denoted by $\Omega_{\tau}$. We write $\Omega_{\tau}^{\prime}:=\cup_{1 \leq t \leq \tau} \Omega_{\tau}$ for the set of all sequences of length at most $\tau$. The

\footnotetext{
${ }^{4}$ This terminology is appropriate in particular for tree models; for instance in binomial models the event set consists of two elements ("up" and "down"). The framework that we use below applies equally well however to models obtained from discretization of a continuous state space, where $A$ would rather be thought of as a representation of a grid in the state space.
} 
collection $\Omega_{T}^{\prime}$ is also written simply as $\Omega^{\prime}$, and we write $\Omega^{\prime \prime}:=\Omega_{T-1}^{\prime}$. Elements of both $\Omega^{\prime}$ and $\Omega^{\prime \prime}$ will be referred to as "partial histories", where the term "partial" should be interpreted in a non-strict sense in the case of $\Omega^{\prime}$ and in a strict sense in the case of $\Omega^{\prime \prime}{ }^{5}$ The length of a sequence $\omega^{\prime} \in \Omega^{\prime}$ is denoted by $\tau\left(\omega^{\prime}\right)$. The set $\Omega_{0}$ of sequences of zero length consists of a single element that we denote by 0 ; this element represents the initial state of the economy. For $\omega=\left(\alpha_{1}, \ldots, \alpha_{T}\right) \in \Omega$ and $1 \leq \tau \leq T$, define the $\tau$-restriction $\left.\omega\right|_{\tau}$ as $\left(\alpha_{1}, \ldots, \alpha_{\tau}\right)$. If a sequence $\omega^{\prime}=\left(\alpha_{1}, \ldots, \alpha_{\tau}\right)$ is a prefix of $\omega \in \Omega$ (i.e. $\left.\omega^{\prime}=\left.\omega\right|_{\tau}\right)$, we write $\omega^{\prime} \preceq \omega$. The collection of all sequences beginning with a given sequence $\omega^{\prime}$ is denoted by $F\left(\omega^{\prime}\right):=\left\{\omega \in \Omega \mid \omega^{\prime} \preceq \omega\right\}$. We denote by $\mathcal{F}_{\tau}$ the algebra generated by the sets $F\left(\omega^{\prime}\right)$ with $\omega^{\prime}$ in the set $\Omega_{\tau}$ of sequences of length exactly $\tau$; in the present setting in which we have a finite sample space, this is of course the same as the $\sigma$-algebra generated by these sets. The collection $\mathcal{F}_{T}$ is the set of all subsets of $\Omega$. The concatenation of a sequence $\omega^{\prime}=\left(\alpha_{1}, \ldots, \alpha_{\tau}\right)$ with an element $\alpha \in A$ is the sequence $\left(\alpha_{1}, \ldots, \alpha_{\tau}, \alpha\right)$, which we write simply as $\omega^{\prime} \alpha$. In this paper, we adopt the conventions $\inf \emptyset=\min \emptyset=\infty$ and $0 \cdot \infty=0 / 0=0$.

\subsubsection{Multiperiod acceptability measures}

Consider a sequence space $\Omega$ as defined in the previous subsection. In the multiperiod setting, the acceptability of a given position should be considered not only as a function of the position itself, but also as a function of available information.

We still define a "position" as a mapping $X$ from $\Omega$ to $\mathbb{R}$. Such a mapping may be restricted to the set $F\left(\omega^{\prime}\right)$ consisting of all sequences beginning with $\omega^{\prime}$. The restricted mapping $\left.X\right|_{F\left(\omega^{\prime}\right)}$ defines a position on $F\left(\omega^{\prime}\right)$. We extend slightly the definition by acceptability measures that was given before by allowing that the degree of acceptability of a given position can be $\infty$. So, an acceptability measure on $F\left(\omega^{\prime}\right)$ is a mapping from $F\left(\omega^{\prime}\right)$ to the extended real line $\mathbb{R} \cup\{\infty\}$.

Definition 2.3 A multiperiod acceptability measure on the sequence space $\Omega$ is a mapping that assigns to each partial history $\omega^{\prime} \in \Omega^{\prime}$ an acceptability measure on $F\left(\omega^{\prime}\right)$.

The acceptability measure on $F\left(\omega^{\prime}\right)$ that is provided by a multiperiod acceptability measure $\phi$ will be denoted by $\phi\left(\cdot \mid \omega^{\prime}\right)$; the element of the extended real line associated by this mapping to a position $X$ on $F\left(\omega^{\prime}\right)$ is denoted by $\phi\left(X \mid \omega^{\prime}\right)$. When $X$ is a position on $\Omega$, we also write $\phi\left(X \mid \omega^{\prime}\right)$ instead of $\phi\left(\left.X\right|_{F\left(\omega^{\prime}\right)} \mid \omega^{\prime}\right)$. The situation at the initial time is represented by the sequence of zero length; instead of $\phi(X \mid 0)$, we write $\phi(X)$. Under the normalization condition (Def. 2.2), we have $\phi(X \mid \omega)=X(\omega)$ for all $\omega \in \Omega$.

\footnotetext{
${ }^{5}$ In the context of a non-recombining tree model, there is a one-one relation between the elements of $\Omega^{\prime}$ and the nodes of the tree. The elements of $\Omega$ correspond to final nodes, and those of $\Omega^{\prime \prime}$ to pre-final nodes.
} 
We say that a multiperiod acceptability measure is coherent if all partial-information acceptability measures $\phi\left(\cdot \mid \omega^{\prime}\right)$ are coherent on $F\left(\omega^{\prime}\right)$. This implies in particular that, for all positions $X$ and $Y$ and for all partial histories $\omega^{\prime}$, the following holds:

$$
\text { if } \phi(X \mid \omega) \geq \phi(Y \mid \omega) \text { for all } \omega \succeq \omega^{\prime} \text {, then } \phi\left(X \mid \omega^{\prime}\right) \geq \phi\left(Y \mid \omega^{\prime}\right) \text {. }
$$

We shall say that a multiperiod acceptability measure satisfies the stepwise monotonicity condition if the following condition holds for all positions $X$ and $Y$ and for all partial histories $\omega^{\prime} \in \Omega^{\prime \prime}:$

$$
\text { if } \phi\left(X \mid \omega^{\prime} \alpha\right) \geq \phi\left(Y \mid \omega^{\prime} \alpha\right) \text { for all } \alpha \in A \text {, then } \phi\left(X \mid \omega^{\prime}\right) \geq \phi\left(Y \mid \omega^{\prime}\right) \text {. }
$$

The example below shows that there exists situations in which the monotonicity property (2.2) is satisfied but the stepwise monotonicity property (2.3) does not hold.

Example 2.4 Consider a two-period binomial tree; that is, let $A=\{u, d\}$ and $\Omega=$ $\{u u, u d, d u, d d\}$. Specify an acceptability measure for products on $\Omega$ by

$$
\phi\left(X \mid \omega^{\prime}\right)=\min _{i=1,2}\left(E_{\mathbb{P}_{i}}\left[X \mid \omega^{\prime}\right]\right)
$$

where $\mathbb{P}_{1}$ is the probability measure that is obtained by assigning probability 0.6 to a $u$ event and 0.4 to a $d$ event, and $\mathbb{P}_{2}$ is obtained by reversing these probabilities. Clearly, $\phi$ is a coherent multiperiod acceptability measure. Consider a position $X$ that pays 100 if $u d$ or $d u$ occurs, and that pays nothing otherwise (a "butterfly"). As is easily computed, we have $\phi(X)=48$ whereas $\phi(X \mid u)=\phi(X \mid d)=40$. Comparing the position $X$ to the position $Y$ that pays 44 in all states of nature, we see that $\phi$ is not stepwise monotonic.

Several authors including Wang (2003) and Epstein and Schneider (2003) use the term "dynamic consistency" for essentially the property that we refer to here as stepwise monotonicity. We prefer to define the notion of dynamic consistency on the basis of a stronger premise, which may make this property easier to establish in some cases. As we show below, the notions of stepwise monotonicity and dynamic consistency (in our sense) are actually the same under the coherence assumption.

Definition 2.5 A multiperiod acceptability measure $\phi$ defined on a sequence space $\Omega$ is said to be dynamically consistent if for all partial histories $\omega^{\prime} \in \Omega^{\prime \prime}$ and all positions $X$ and $Y$ we have

$$
\text { if } \phi\left(X \mid \omega^{\prime} \alpha\right)=\phi\left(Y \mid \omega^{\prime} \alpha\right) \text { for all } \alpha \in A \text {, then } \phi\left(X \mid \omega^{\prime}\right)=\phi\left(Y \mid \omega^{\prime}\right) \text {. }
$$

Proposition 2.6 A coherent multiperiod acceptability measure is dynamically consistent if and only if it is stepwise monotonic. 
ProOF It is immediate that stepwise monotonicity implies dynamic consistency, even without the coherence assumption. To show the converse, let $\phi$ be a coherent and dynamically consistent acceptability measure, and suppose that the positions $X$ and $Y$ and the partial history $\omega^{\prime} \in \Omega^{\prime \prime}$ are such that $\phi\left(X \mid \omega^{\prime} \alpha\right) \geq \phi\left(Y \mid \omega^{\prime} \alpha\right)$ for all $\alpha \in A$. Define a position $\tilde{X}$ on $F\left(\omega^{\prime}\right)$ by $\tilde{X}(\omega)=\phi\left(X \mid \omega^{\prime} \alpha\right)$ if $\omega \succeq \omega^{\prime} \alpha$, and define $\tilde{Y}$ similarly. Because $\phi\left(\cdot \mid \omega^{\prime} \alpha\right)$ is normalized, we have $\phi\left(\tilde{X} \mid \omega^{\prime} \alpha\right)=\phi\left(X \mid \omega^{\prime} \alpha\right)$ for all $\alpha \in A$, and therefore, by dynamic consistency, $\phi\left(\tilde{X} \mid \omega^{\prime}\right)=\phi\left(X \mid \omega^{\prime}\right)$. In the same way, it follows that $\phi\left(\tilde{Y} \mid \omega^{\prime}\right)=\phi\left(Y \mid \omega^{\prime}\right)$. Moreover, we have $\tilde{X}(\omega) \geq \tilde{Y}(\omega)$ for all $\omega \succeq \omega^{\prime}$, which shows, by the monotonicity property of $\phi\left(\cdot \mid \omega^{\prime}\right)$, that $\phi\left(\tilde{X} \mid \omega^{\prime}\right) \geq \phi\left(\tilde{Y} \mid \omega^{\prime}\right)$. It follows that $\phi\left(X \mid \omega^{\prime}\right) \geq \phi\left(Y \mid \omega^{\prime}\right)$.

We introduce some further notation and terminology that will be needed below. To each $\omega^{\prime} \in \Omega^{\prime \prime}$, one can associate a single-period economy in which the events that may occur (equivalently, the states of nature that may arise after one time step) are parametrized by the event set $A$. A single-period position is a mapping from $A$ to $\mathbb{R}$. A single-period acceptability measure assigns real numbers to single-period positions.

Let a multiperiod acceptability measure $\phi$ be given. For any partial history $\omega^{\prime} \in \Omega^{\prime \prime}$, one can generate a position $X_{Y}$ on $F\left(\omega^{\prime}\right)$ from a given single-period position $Y: A \rightarrow \mathbb{R}$ by defining

$$
X_{Y}(\omega)=Y(\alpha) \text { if } \omega \succeq \omega^{\prime} \alpha .
$$

In this way we can introduce for each $\omega^{\prime} \in \Omega^{\prime \prime}$ a single-period acceptability measure denoted by $\phi_{\omega^{\prime}}$ :

$$
\phi_{\omega^{\prime}}: Y \mapsto \phi\left(X_{Y} \mid \omega^{\prime}\right) .
$$

The following lemma is easily verified directly from the coherence axioms.

LEMMA 2.7 If $\phi$ is a coherent multiperiod acceptability measure, then all single-period acceptability measures $\phi_{\omega^{\prime}}$ derived from $\phi$ are coherent as well.

Given a product $X$ on the sequence space $\Omega$ and a multiperiod acceptability measure $\phi$, we can define for each partial history a single-period position $\phi\left(X \mid \omega^{\prime} \cdot\right)$ in the following way:

$$
\phi\left(X \mid \omega^{\prime} \cdot\right): \alpha \mapsto \phi\left(X \mid \omega^{\prime} \alpha\right)
$$

Since this is a single-period position, its acceptability may be evaluated by means of the single-period acceptability measure $\phi_{\omega^{\prime}}$. If $\phi$ is dynamically consistent, we have

$$
\phi\left(X \mid \omega^{\prime}\right)=\phi_{\omega^{\prime}}\left(\phi\left(X \mid \omega^{\prime} \cdot\right)\right) .
$$


This implies that the multiperiod acceptability measure $\phi$ can be described in terms of the conditional single-period acceptability measures $\phi_{\omega^{\prime}}$.

An obvious way to construct a dynamically consistent acceptability measure is to start by assigning a single-period acceptability measure $\phi^{\omega^{\prime}}$ to each partial history $\omega^{\prime} \in \Omega^{\prime \prime}$ (for instance one may use the same acceptability measure for each $\left.\omega^{\prime}\right)$ and then to define $\phi\left(\cdot \mid \omega^{\prime}\right)$ recursively by

$$
\begin{array}{rlrl}
\phi(X \mid \omega) & =X(\omega) & & (\omega \in \Omega) \\
\phi\left(X \mid \omega^{\prime}\right) & =\phi^{\omega^{\prime}}\left(\phi\left(X \mid \omega^{\prime} \cdot\right)\right) & \left(\omega^{\prime} \in \Omega^{\prime \prime}\right) .
\end{array}
$$

In the following lemma we verify some properties of this scheme. Extending the terminology introduced in Def. 2.2, we say that a multiperiod acceptability measure $\phi$ is normalized if each measure $\phi\left(\cdot \mid \omega^{\prime}\right)$ is a normalized acceptability measure on $F\left(\omega^{\prime}\right)$.

Lemma 2.8 The acceptability measure $\phi$ that is defined on the sequence space $\Omega$ from a family $\left\{\phi^{\omega^{\prime}}\right\}_{\omega^{\prime} \in \Omega^{\prime \prime}}$ via the rule (2.10) is normalized if all measures $\phi^{\omega^{\prime}}$ are normalized, and in this case we have $\phi_{\omega^{\prime}}=\phi^{\omega^{\prime}}$ for all $\omega^{\prime}$. Moreover, if all $\phi^{\omega^{\prime}}$ are coherent, then $\phi$ is coherent as well.

Proof The first claim follows easily by induction. To show that $\phi_{\omega^{\prime}}=\phi^{\omega^{\prime}}$, take a singleperiod position $Y: A \rightarrow \mathbb{R}$, and let $X_{Y}$ be defined as in (2.6). Due to the normalization property we have $\phi\left(X_{Y} \mid \omega^{\prime} \alpha\right)=Y(\alpha)$ for all $\alpha \in A$, and so

$$
\phi_{\omega^{\prime}}(Y)=\phi\left(X_{Y} \mid \omega^{\prime}\right)=\phi^{\omega^{\prime}}\left(\phi\left(X_{Y} \mid \omega^{\prime} \cdot\right)\right)=\phi^{\omega^{\prime}}(Y)
$$

Since this holds for all $Y$, it follows that $\phi_{\omega^{\prime}}=\phi^{\omega^{\prime}}$. Finally, the fact that coherence of $\phi$ follows from coherence of all $\phi^{\omega^{\prime}}$ can be verified by induction directly from the coherence axioms.

It follows from the lemma that any dynamically consistent acceptability measure can be thought of as having been constructed from single-period acceptability measures by means of the rule (2.10). For coherent risk measures, we can ask what this means in terms of the representing collection of probability measures. This question is answered below.

\subsubsection{Representation by collections of probability measures}

A probability measure $\mathbb{P}$ on $\left(\Omega, \mathcal{F}_{T}\right)$ can be defined in a straightforward way by assigning a probability to each trajectory $\omega$. With slight abuse of notation, we denote the probability that $\omega$ will occur by $\mathbb{P}(\omega)$. The marginal probability of a sequence $\omega^{\prime} \in \Omega^{\prime}$ is given, with some further abuse of notation, by

$$
\mathbb{P}\left(\omega^{\prime}\right)=\sum_{\omega^{\prime} \preceq \omega} \mathbb{P}(\omega)=\mathbb{P}\left(F\left(\omega^{\prime}\right)\right) .
$$


The conditional probability given a sequence $\omega^{\prime} \in \Omega^{\prime}$ of a sequence $\omega \succeq \omega^{\prime}$ is given by

$$
\mathbb{P}\left(\omega \mid \omega^{\prime}\right)=\frac{\mathbb{P}(\omega)}{\mathbb{P}\left(\omega^{\prime}\right)}
$$

We will also need the "single-period conditional probabilities" defined by

$$
\mathbb{P}^{\mathrm{S}}\left(\alpha \mid \omega^{\prime}\right)=\frac{\mathbb{P}\left(\omega^{\prime} \alpha\right)}{\mathbb{P}\left(\omega^{\prime}\right)} .
$$

For future reference we note the decomposition with respect to single-step marginals

$$
\mathbb{P}\left(\omega \mid \omega^{\prime}\right)=\mathbb{P}^{\mathrm{s}}\left(\alpha \mid \omega^{\prime}\right) \mathbb{P}\left(\omega \mid \omega^{\prime} \alpha\right) \quad \text { if } \omega^{\prime} \alpha \preceq \omega
$$

and the law of iterated expectations

$$
\begin{aligned}
E\left[X \mid \omega^{\prime}\right] & =\sum_{\omega \succeq \omega^{\prime}} \mathbb{P}\left(\omega \mid \omega^{\prime}\right) X(\omega) \\
& =\sum_{\alpha \in A} \mathbb{P}^{\mathrm{s}}\left(\alpha \mid \omega^{\prime}\right) \sum_{\omega \succeq \omega^{\prime} \alpha} \mathbb{P}\left(\omega \mid \omega^{\prime} \alpha\right) X(\omega) \\
& =E_{\mathbb{P}^{\mathrm{S}}\left(\cdot \mid \omega^{\prime}\right)} E\left[X \mid \omega^{\prime} \alpha\right] .
\end{aligned}
$$

Given a collection $\mathcal{P}$ of probability measures on $\Omega$, we can define a multiperiod acceptability measure $\phi_{\mathcal{P}}$ by defining

$$
\phi_{\mathcal{P}}\left(X \mid \omega^{\prime}\right)=\inf _{\mathbb{P} \in \mathcal{P}, \mathbb{P}\left(\omega^{\prime}\right)>0} E_{\mathbb{P}}\left[X \mid \omega^{\prime}\right]
$$

for positions $X$ and partial histories $\omega^{\prime}$. To avoid having to deal with trivial exceptions we introduce as a standing assumption that risk measures satisfy an axiom of relevance (cf. Artzner et al. (1999; p. 210) and Föllmer and Schied (2002c, p. 347)).

Assumption 2.9 If $\phi$ denotes a multiperiod acceptability measure, then, for all $\omega^{\prime} \in \Omega^{\prime}$,

$$
X \leq 0 \text { and } X \neq 0 \text { implies } \phi\left(X \mid \omega^{\prime}\right)<0 .
$$

In other words, we assume that in (2.16) the union of supports of measures in $\mathcal{P}$ equals $\Omega .^{6}$

It is easily seen that a coherent acceptability measure is representable in the form (2.16) if and only if the positions $X$ that are acceptable given a partial history $\omega^{\prime}$ are exactly those for which the position $X \cdot \mathbb{1}_{F\left(\omega^{\prime}\right)}$ is acceptable at the initial time. Clearly, a multiperiod acceptability measure need not satisfy this property even if it is coherent, since our general definition implies no relations between the conditional acceptability measures related to different partial histories. Multiperiod acceptability measures of the form (2.16) might be

\footnotetext{
${ }^{6}$ See Artzner et al. (1999; remark 4.3) for a proof. Note that finiteness of $\phi_{\mathcal{P}}$ in (2.16) is equivalent to a weaker form of relevance, corresponding to the condition that the union of supports of test measures contains $\Omega^{\prime \prime}$.
} 
called completely coherent. Example 2.4 shows that a completely coherent measure is not necessarily dynamically consistent. It will follow from Thm. 2.12 below that the reverse implication does hold true.

If a collection $\mathcal{P}$ of probability measures on $\Omega$ is given, one can define for each partial history $\omega^{\prime} \in \Omega^{\prime \prime}$ a collection of single-period probability measures by

$$
\mathcal{P}^{\mathrm{s}}\left(\omega^{\prime}\right)=\left\{\mathbb{P}^{\mathrm{s}}\left(\cdot \mid \omega^{\prime}\right) \mid \mathbb{P} \in \mathcal{P} \text { with } \mathbb{P}\left(\omega^{\prime}\right)>0\right\}
$$

where $\mathbb{P}^{\mathrm{s}}\left(\cdot \mid \omega^{\prime}\right)$ is defined by (2.13). Conversely, if for each $\omega^{\prime} \in \Omega^{\prime \prime}$ a collection of singleperiod measures $\mathcal{P}_{\mathrm{S}}\left(\omega^{\prime}\right)$ is given, then the family $\left\{\mathcal{P}_{\mathrm{S}}\left(\omega^{\prime}\right)\right\} \omega_{\omega^{\prime} \in \Omega^{\prime \prime}}$ defines a collection of probability measures on $\Omega$ by

$$
\mathcal{P}=\left\{\mathbb{P} \mid \mathbb{P}^{\mathrm{s}}\left(\cdot \mid \omega^{\prime}\right) \in \mathcal{P}_{\mathrm{s}}\left(\omega^{\prime}\right) \text { for all } \omega^{\prime} \in \Omega^{\prime \prime} \text { s.t. } \mathbb{P}\left(\omega^{\prime}\right)>0\right\}
$$

If the above relation holds, we say that the collection $\mathcal{P}$ is generated by the family $\left\{\mathcal{P}_{\mathrm{S}}\left(\omega^{\prime}\right)\right\}_{\omega^{\prime} \in \Omega^{\prime \prime}}$. In more concrete terms, the generated probability measures are of the form

$$
\mathbb{P}:\left(\alpha_{1}, \ldots, \alpha_{T}\right) \mapsto \prod_{t=1}^{T} \mathbb{P}_{t}^{\mathrm{S}}\left(\alpha_{t}\right)
$$

where for each $t$

$$
\mathbb{P}_{t}^{\mathrm{s}} \in \mathcal{P}_{\mathrm{s}}\left(\left(\alpha_{1}, \ldots, \alpha_{t-1}\right)\right) \text {. }
$$

Starting with a given collection of probability measures $\mathcal{P}$ we can first form its associated family of collections of single-period measures, and then form the collection $\mathcal{P}^{\prime}$ of measures generated by this family. It may well happen that the collection $\mathcal{P}^{\prime}$ obtained in this way is larger than the original collection $\mathcal{P}$; see for instance Example 2.4. In the following definition we introduce a term for collections of probability measures that do not change under the operation just described.

Definition 2.10 A collection of probability measures $\mathcal{P}$ on a sequence space $\Omega$ is said to be of product type if

$$
\mathcal{P}=\left\{\mathbb{P} \mid \mathbb{P}^{\mathrm{s}}\left(\cdot \mid \omega^{\prime}\right) \in \mathcal{P}^{\mathrm{s}}\left(\omega^{\prime}\right) \text { for all } \omega^{\prime} \in \Omega^{\prime \prime} \text { s. t. } \mathbb{P}\left(\omega^{\prime}\right)>0\right\} .
$$

Example 2.4 has shown that a multiperiod acceptability measure specified by a collection of test measures via (2.16) is not necessarily dynamically consistent. The following lemma will be used below to prove that dynamic consistency does hold for acceptability measures obtained from product-type collections of probability measures.

LEMMA 2.11 Let $\mathcal{P}$ be a product-type collection of test measures, with generating family $\left\{\mathcal{P}^{\mathrm{s}}\left(\omega^{\prime}\right)\right\}_{\omega^{\prime} \in \Omega^{\prime \prime}}$, and let the associated acceptability measure be denoted by $\phi$. For any product $X$ and any partial history $\omega^{\prime} \in \Omega^{\prime \prime}$, we have

$$
\phi\left(X \mid \omega^{\prime}\right)=\inf _{P \in \mathcal{P}^{\mathrm{s}}\left(\omega^{\prime}\right)} E_{P} \phi\left(X \mid \omega^{\prime} \alpha\right) .
$$


Proof Take a partial history $\omega^{\prime}$, a position $X$, and a test measure $\mathbb{P} \in \mathcal{P}$. By the law of iterated expectations, we have

$$
\begin{aligned}
E_{\mathbb{P}}\left[X \mid \omega^{\prime}\right] & =E_{\mathbb{P}^{S}\left(\cdot \mid \omega^{\prime}\right)} E_{\mathbb{P}}\left[X \mid \omega^{\prime} \alpha\right] \\
& \geq E_{\mathbb{P}^{S}\left(\cdot \mid \omega^{\prime}\right)} \inf _{\mathbb{P} \in \mathcal{P}} E_{\mathbb{P}}\left[X \mid \omega^{\prime} \alpha\right] \\
& =E_{\mathbb{P}^{S}\left(\cdot \mid \omega^{\prime}\right)} \phi\left(X \mid \omega^{\prime} \alpha\right) \\
& \geq \inf _{P \in \mathcal{P}^{S}\left(\omega^{\prime}\right)} E_{P} \phi\left(X \mid \omega^{\prime} \alpha\right) .
\end{aligned}
$$

Since this holds for all $\mathbb{P} \in \mathcal{P}$, it follows that

$$
\phi\left(X \mid \omega^{\prime}\right)=\inf _{\mathbb{P} \in \mathcal{P}} E_{\mathbb{P}}\left[X \mid \omega^{\prime}\right] \geq \inf _{P \in \mathcal{P}^{\mathrm{s}}\left(\omega^{\prime}\right)} E_{P} \phi\left(X \mid \omega^{\prime} \alpha\right) .
$$

To show the reverse inequality, take $\varepsilon>0$ and let $P \in \mathcal{P}^{\mathrm{s}}\left(\omega^{\prime}\right)$. Because $\phi\left(X \mid \omega^{\prime} \alpha\right)=$ $\inf _{\mathbb{P} \in \mathcal{P}} E_{\mathbb{P}}\left[X \mid \omega^{\prime} \alpha\right]$, we can choose for each $\alpha$ a probability measure $\mathbb{P}_{\alpha} \in \mathcal{P}$ such that

$$
E_{\mathbb{P}_{\alpha}}\left[X \mid \omega^{\prime} \alpha\right] \leq \phi\left(X \mid \omega^{\prime} \alpha\right)+\varepsilon .
$$

By the assumption that the collection $\mathcal{P}$ is of product type, there exists a probability measure $\mathbb{P} \in \mathcal{P}$ such that $\mathbb{P}^{s}\left(\cdot \mid \omega^{\prime}\right)=P$ and $\mathbb{P}\left(\cdot \mid \omega^{\prime} \alpha\right)=\mathbb{P}_{\alpha}\left(\cdot \mid \omega^{\prime} \alpha\right)$ for all $\alpha \in A$. Then

$$
\begin{aligned}
E_{\mathbb{P}}\left[X \mid \omega^{\prime}\right] & =E_{\mathbb{P}^{\mathrm{s}}\left(\cdot \mid \omega^{\prime}\right)} E_{\mathbb{P}}\left[X \mid \omega^{\prime} \alpha\right] \\
& =E_{P} E_{\mathbb{P}_{\alpha}}\left[X \mid \omega^{\prime} \alpha\right] \\
& \leq E_{P}\left(\phi\left(X \mid \omega^{\prime} \alpha\right)+\varepsilon\right) \\
& =E_{P} \phi\left(X \mid \omega^{\prime} \alpha\right)+\varepsilon .
\end{aligned}
$$

It follows that

$$
E_{P} \phi\left(X \mid \omega^{\prime} \alpha\right) \geq E_{\mathbb{P}}\left[X \mid \omega^{\prime}\right]-\varepsilon \geq \phi\left(X \mid \omega^{\prime}\right)-\varepsilon .
$$

Since this holds for all positive $\varepsilon$, we obtain $E_{P} \phi\left(X \mid \omega^{\prime} \alpha\right) \geq \phi\left(X \mid \omega^{\prime}\right)$, and since $P \in \mathcal{P}^{\mathrm{s}}\left(\omega^{\prime}\right)$ was arbitrary, it follows that

$$
\inf _{P \in \mathcal{P}^{\mathrm{s}}\left(\omega^{\prime}\right)} E_{P} \phi\left(X \mid \omega^{\prime} \alpha\right) \geq \phi\left(X \mid \omega^{\prime}\right) .
$$

This completes the proof.

We now show that, for coherent multiperiod acceptability measures, the properties of dynamic consistency and of representability by a product-type collection are in fact equivalent.

ThEOREM 2.12 A coherent multiperiod acceptability measure is dynamically consistent if and only if it can be represented, via relation (2.16), by a product-type collection of probability measures. 
Proof Let a multiperiod acceptability measure $\phi$ be defined by the relation (2.16) where the collection $\mathcal{P}$ is of product type. The preceding lemma shows that, for each position $X$ and each $\omega^{\prime} \in \Omega^{\prime \prime}$, the degree of acceptability $\phi\left(X \mid \omega^{\prime}\right)$ is determined in terms of the values of $\phi\left(X \mid \omega^{\prime} \alpha\right)$, and it follows immediately that the acceptability measure $\phi$ is dynamically consistent.

Conversely, let $\phi$ be a dynamically consistent and coherent acceptability measure. As noted in Lemma 2.7, the single-period acceptability measures $\phi_{\omega^{\prime}}$ derived from $\phi$ are all coherent, and so by the representation result Thm. 2.1 there exists for each $\omega^{\prime} \in \Omega^{\prime \prime}$ a collection $\mathcal{P}_{\mathrm{s}}\left(\omega^{\prime}\right)$ of probability measures on the event set $A$ such that $\phi_{\omega^{\prime}}(Y)=\inf _{P \in \mathcal{P}_{\mathrm{s}}\left(\omega^{\prime}\right)} E_{P} Y$ for any single-period position $Y$. Let $\mathcal{P}$ denote the product-type collection of probability measures on $\Omega$ that is generated by the family $\left\{\mathcal{P}_{\mathbf{s}}\left(\omega^{\prime}\right)\right\}_{\omega^{\prime} \in \Omega^{\prime \prime}}$ (notice that $\mathcal{P}^{\mathrm{s}}\left(\omega^{\prime}\right)$ is non-empty because of Assumption 2.9). For any position $X$, we have

$$
\begin{aligned}
\phi\left(X \mid \omega^{\prime}\right) & =\phi_{\omega^{\prime}}\left(\phi\left(X \mid \omega^{\prime} \cdot\right)\right) \\
& =\inf _{P \in \mathcal{P}_{\mathrm{S}}\left(\omega^{\prime}\right)} E_{P} \phi\left(X \mid \omega^{\prime} \alpha\right) \\
& =\phi_{\mathcal{P}}\left(X \mid \omega^{\prime}\right)
\end{aligned}
$$

where we used the preceding lemma in the final equality. This shows that $\phi$ is represented by the product-type collection $\mathcal{P}$.

\section{Pricing in incomplete markets}

The main purpose of this section is to obtain price bounds for derivative products in incomplete markets. For this purpose we suppose that an acceptability measure (i.e. a collection of test measures) has been selected in a sufficiently conservative way so that, in a straightforward extension of the standard arbitrage argument, any opportunity that is acceptable according this measure would be quickly eliminated in the market. After this adjustment has taken place, the price of any asset must be such that it is not possible to create acceptable opportunities by means of any admissible portfolio strategy. We aim to compute the resulting price bounds explicitly.

\subsection{Absence of strictly acceptable opportunities}

We assume that $n$ basic assets are present in the market, whose prices are described by a

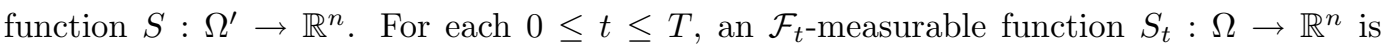
defined by $S_{t}(\omega)=S\left(\left.\omega\right|_{t}\right)$. A trading strategy is a function from $\Omega^{\prime}$ to $\mathbb{R}^{n}$, interpreted as a rule that assigns to each partial history $\omega^{\prime} \in \Omega^{\prime}$ a position in the basic instruments. Again, if 
$g: \Omega^{\prime} \rightarrow \mathbb{R}^{n}$ is a strategy, we write $g_{t}(\omega)=g\left(\left.\omega\right|_{t}\right)$. Each trading strategy defines a position, namely the total result of the strategy which is given by

$$
H^{g}:=\sum_{t=0}^{T-1} g_{t}^{\top}\left(S_{t+1}-S_{t}\right)
$$

for a self-financing strategy with zero initial investment. Given a basic acceptability measure $\phi$, we define the acceptability measure of a position $X$ subject to a strategy $g$ by

$$
\phi^{g}(X):=\phi\left(X+H^{g}\right) .
$$

Let us assume that a nonempty set $\mathbb{G}$ of allowed hedging strategies has been fixed. We can then define, for any position $X$, the optimal degree of acceptability taking hedging into account:

$$
\phi^{*}(X):=\sup _{g \in \mathbb{G}} \phi^{g}(X) .
$$

In general the supremum need not be finite. If $\phi^{*}(X)=\infty$ then arbitrarily high degrees of acceptability can be achieved, which may not seem realistic at least in some interpretations of acceptability measures. Therefore we are interested in conditions that ensure finiteness of the supremum in (3.3).

Consider first, as in Carr et al. (2001), a single-period economy with traded assets $S^{0}, \ldots, S^{n}$ and with a collection $\mathcal{P}$ of probability measures on the finite set $\Omega$ of states of nature. The price of asset $i$ at time $t(t=0,1)$ is given by $S_{t}^{i} ; S^{0}$ is the numéraire which always has price 1 . The economy is said to allow strictly acceptable opportunities if it is possible to form a strictly acceptable portfolio at zero cost; that is, if there exist portfolio weights $a_{0}, \ldots, a_{n}$ such that

$$
\begin{aligned}
\sum_{i=0}^{n} a_{i} S_{0}^{i} & =0 \\
E_{\mathbb{P}} \sum_{i=0}^{n} a_{i} S_{1}^{i} & \geq 0 \quad \text { for all } \mathbb{P} \in \mathcal{P} \\
E_{\mathbb{P}} \sum_{i=0}^{n} a_{i} S_{1}^{i} & >0 \quad \text { for some } \mathbb{P} \in \mathcal{P} .
\end{aligned}
$$

Carr et al. (2001) have argued that if a collection of test measures is chosen sufficiently large so as to reflect a widely held market view, it can be assumed that there will be no strictly acceptable opportunities in the economy. The NSAO condition ("no strictly acceptable opportunities") is a stronger requirement than absence of arbitrage ${ }^{7}$, and in incomplete markets it therefore leads in general to tighter bounds on prices of contingent claims than would be obtained by the no-arbitrage condition alone.

In a multiperiod setting, we interpret the NSAO condition as the requirement that no self-financing investment strategy with zero initial cost should produce a strictly acceptable

\footnotetext{
${ }^{7}$ Notice that Assumption 2.9 guarantees that no arbitrage opportunity can be overlooked by every test.
} 
result. It is easily verified that a necessary condition for the NSAO condition to hold for a given multiperiod economy is that each of the associated single-period economies should be free of strictly acceptable opportunities. As can be seen from simple examples, however, this condition is not sufficient.

Example 3.1 Consider the two-period binomial tree of Example 2.4 again, with the same collection of two test measures. Suppose there are two assets $S$ and $B$. The value of $B$ is always 100 , whereas for $S$ we have

$$
\begin{gathered}
S(0)=100, \quad S(u)=110, \quad S(d)=90, \\
S(u u)=120, \quad S(u d)=S(d u)=100, \quad S(d d)=80 .
\end{gathered}
$$

It is easily verified that none of the single-period economies derived from this model allows strictly acceptable opportunities. Now consider the dynamic strategy that is defined as follows. Take no position at the initial time; at time 1, take a position 1 in the asset $S$ (and -1.1 in $B$ ) if an "up" movement occurs, and take the opposite position (and 0.9 in $B$ ) if a "down" step takes place. The expected result of this strategy under test measure $\mathbb{P}_{1}$ is

$$
0.6 \cdot(0.6 \cdot 10+0.4 \cdot(-10))+0.4 \cdot(0.6 \cdot(-10)+0.4 \cdot 10)=0.4
$$

while under $\mathbb{P}_{2}$ we find

$$
0.4 \cdot(0.4 \cdot 10+0.6 \cdot(-10))+0.6 \cdot(0.4 \cdot(-10)+0.6 \cdot 10)=0.4 \text {. }
$$

So the expected result is positive in both cases; the "momentum" strategy creates a strictly acceptable opportunity.

In the example, the collection of test measures is not large enough to counterbalance the flexibility of dynamic strategies. An obvious way to extend the set of test measures is to form the product-type collection generated by the single-period probability measures that are implied by the two original test measures; this is the procedure already suggested just before Def. 2.10. For instance, this would generate a measure that assigns probability 0.6 to an "up" movement in the first step but probability 0.4 to the same movement at the second step, conditional on occurrence of an upward movement on the first step. It can easily be seen that the set of eight probability measures obtained in this way is sufficiently large to eliminate all strictly acceptable opportunities in the example economy.

A necessary condition for the multiperiod NSAO condition to hold is that each of the single-period economies satisfies the NSAO property. Under the assumption of dynamic consistency, this condition is sufficient as well, as shown in the theorem below. 
Theorem 3.2 Consider a multiperiod economy with assets $S^{1}, \ldots, S^{n}$ and a collection $\mathcal{P}$ of test measures. If the collection $\mathcal{P}$ is of product type, and if no single-period economy allows a strictly acceptable opportunity, then the multiperiod economy allows no strictly acceptable opportunities.

The proof of the theorem is given in the appendix. It has been shown by Carr et al. in a single-period situation, and under the assumption that the set of test measures is finite ${ }^{8}$ that absence of strictly acceptable opportunities is equivalent to the existence of a representative state pricing function which expresses the prices of Arrow-Debreu securities as expected values under a certain linear combination, with strictly positive coefficients, of test measures. It follows from the above result that, under dynamic consistency, this property can be generalized to the multiperiod situation.

REMARK 3.3 In Thm. 3.2, dynamic consistency is a sufficient but not a necessary condition for absence of strictly acceptable opportunities in the multiperiod economy. It may be verified that in Example 3.1 the NSAO condition can also be obtained by adding just one test measure, for instance the measure $\mathbb{P}_{3}$ that assigns probabilities $0.24,0.26,0.26$, and 0.24 to the events $u u, u d, d u$, and $d d$, respectively. Product-type completion of the two given measures leads to the addition of six test measures. So the theorem above provides a way of guaranteeing NSAO that is systematic, but possibly conservative. Fulfillment of the multiperiod NSAO property is already achieved by the addition of any test measures such that there is a nontrivial convex combination of the extended collection of test measures that produces a martingale. In the numerical example given before, note that $\frac{1}{4} \mathbb{P}_{1}+\frac{1}{4} \mathbb{P}_{2}+\frac{1}{2} \mathbb{P}_{3}$ is a martingale measure (which is in fact unique in this example). It may also be noted that the anomaly of Example 2.4 is not cured by the addition of the test measure $\mathbb{P}_{3}$.

\subsection{Recursive Formulation of Price Bounds}

Given a product $X$ and a set of admissible hedging strategies $\mathbb{G}$, the degree of acceptability of $X$ at time 0 under optimal hedging has been defined as

$$
\phi^{*}(X)=\sup _{g \in \mathbb{G}} \phi\left(X+H^{g}\right) .
$$

In Jaschke and Küchler (2001; Proposition 7), it is shown that if $\phi$ is coherent, and $\mathbb{G}$ is a cone, then $\phi^{*}$ is coherent as well. In this way one obtains coherent valuation bounds for $X$ : a price for $X$ outside the interval $\left[\phi^{*}(X),-\phi^{*}(-X)\right]$ would induce a strictly acceptable opportunity consisting of a suitably hedged long or short position in $X$. We will be interested below in obtaining recursive schemes to compute these bounds.

\footnotetext{
${ }^{8}$ Equivalently, it may be assumed that the set of test measures is finitely generated, i.e. there exists a finite set of measures such that each test measure can be written as a convex combination of these.
} 
It is possible to characterize the transformed family $\mathcal{P}^{*}$ that represents $\phi^{*}$, in the sense that $\phi_{\mathcal{P}}^{*}=\phi_{\mathcal{P}^{*}}$; see Föllmer and Schied (2002a, 2002c) for results in a more general setting. Our main interest in this section is to obtain recursive schemes for the computation of the optimal degree of acceptability under hedging.

For the purposes of the recursion, we define for a given hedging strategy $g$ :

$$
H_{\tau}^{g}(\omega)=\sum_{t=\tau}^{T-1} g_{t}^{\top}(\omega)\left(S_{t+1}(\omega)-S_{t}(\omega)\right) \quad(\omega \in \Omega) .
$$

Note that the lower limit of the summation in (3.5) is $t=\tau$. In keeping with the standard convention that assigns the value 0 to a sum with no terms, we set

$$
H_{T}^{g}(\omega)=0
$$

for all histories $\omega$ and all strategies $g$. For a given product $X$ we define

$$
\phi^{g}\left(X \mid \omega^{\prime}\right)=\phi\left(X+H_{\tau\left(\omega^{\prime}\right)}^{g} \mid \omega^{\prime}\right)
$$

and

$$
\phi^{*}\left(X \mid \omega^{\prime}\right)=\sup _{g \in \mathbb{G}} \phi^{g}\left(X \mid \omega^{\prime}\right) .
$$

The following theorem provides a recursive max-min characterization of the optimal degree of acceptability. The proof of the theorem is provided in the appendix.

Theorem 3.4 Consider an economy with asset prices $S^{i}: \Omega^{\prime} \rightarrow \mathbb{R}(i=1, \ldots, n)$ and with a collection $\mathcal{P}$ of test measures that is of product type. Let the collection $\mathcal{P}$ be generated by the family $\left\{\mathbb{P}^{s}\left(\omega^{\prime}\right)\right\}_{\omega^{\prime} \in \Omega^{\prime \prime}}$. Assume that a set of admissible hedge strategies $\mathbb{G}$ is given consisting of all functions from $\Omega^{\prime}$ to $G$ where $G \subset \mathbb{R}^{n}$. For any product $X$ and any partial history $\omega^{\prime} \in \Omega^{\prime \prime}$, we have

$$
\phi^{*}\left(X \mid \omega^{\prime}\right)=\sup _{\gamma \in G} \inf _{P \in \mathcal{P}^{\mathrm{s}}\left(\omega^{\prime}\right)} E_{P}\left[\phi^{*}\left(X \mid \omega^{\prime} \alpha\right)+\gamma^{\top}\left(S\left(\omega^{\prime} \alpha\right)-S\left(\omega^{\prime}\right)\right)\right] .
$$

If for all $\omega^{\prime} \in \Omega^{\prime}$ the supremum in (3.8) is achieved, say in $\gamma\left(\omega^{\prime}\right)$, then the strategy $g^{*}$ defined by

$$
g^{*}: \omega^{\prime} \mapsto \gamma\left(\omega^{\prime}\right) \quad\left(\omega^{\prime} \in \Omega^{\prime \prime}\right)
$$

optimizes the degree of acceptability of the position $X$, i.e., for all $\omega^{\prime} \in \Omega^{\prime}$,

$$
\phi^{*}\left(X \mid \omega^{\prime}\right)=\phi^{g^{*}}\left(X \mid \omega^{\prime}\right) .
$$

The theorem does not claim that there is a unique strategy that optimizes acceptability. It may well happen that at one or more partial histories $\omega^{\prime}$ there are several different values of $\gamma$ that achieve the supremum in (3.8). In this case there are several different strategies that reach the same optimal level of acceptability. 
In the above, we have allowed restrictions of hedging positions so that for instance shortselling constraints can be accommodated. These restrictions could be made state-dependent at the cost of just a bit more complex notation. If hedging positions are unrestricted, on the other hand, then the max-min characterization can be considerably simplified. The following implication of Proposition 20 of Föllmer and Schied (2002a), which in fact does not involve dynamic consistency at all, deserves to be made explicit in this context. A derivation is given in the appendix. The proposition below can also be derived from the version of the fundamental theorem of asset pricing due to Jaschke and Küchler (2001, Cor. 8).

Proposition 3.5 Let $\mathcal{P}$ be a collection of test measures representing an acceptability measure $\phi$ for a multiperiod economy given by an asset price process $S$. Under the assumption of unrestricted trading $\left(G=\mathbb{R}^{n}\right)$, the optimal acceptability measure $\phi^{*}$ in (3.4) is represented by the set $\mathcal{P}_{0}$ of all measures in the closed convex hull of $\mathcal{P}$ under which the price process $S$ is a martingale.

We hence may write $\phi_{\mathcal{P}}^{*}=\phi_{\mathcal{P}_{0}}$. By combining this with Theorem 3.4, the max-min problem in (3.8) can simplified considerably.

Corollary 3.6 Assume the setting of Prop. 3.5, with $\mathcal{P}_{0}$ of product type. Let the collection of single-step martingale measures in $\mathcal{P}^{\mathrm{s}}\left(\omega^{\prime}\right)$ be denoted by $\mathcal{P}_{0}^{\mathrm{s}}\left(\omega^{\prime}\right)$. If $\mathcal{P}_{0}^{\mathrm{s}}\left(\omega^{\prime}\right)$ is not empty, then

$$
\phi^{*}\left(X \mid \omega^{\prime}\right)=\inf _{P \in \mathcal{P}_{0}^{\mathbf{S}}\left(\omega^{\prime}\right)} E_{P} \phi^{*}\left(X \mid \omega^{\prime} \alpha\right) .
$$

So, under unrestricted trading, optimal hedging reduces the set of test measures to the set of its risk-neutral elements, whose evaluations are not affected by trading. If the remaining collection of risk-neutral measures is dynamically consistent, the backward recursion in (2.22) for acceptability can be applied in terms of price bounds. When the set $\mathcal{P}_{0}^{\mathrm{s}}\left(\omega^{\prime}\right)$ over which minimization takes place is empty, the relation (3.10) still holds on the basis of the convention that the minimum over an empty set is $\infty$; in this case, the NSAO condition is not satisfied.

\section{The axiom of dynamic consistency}

In the literature on choice under uncertainty in multiperiod situations, dynamic consistency appears to be widely accepted as an aspect of rational behavior. For instance, Barberis et al. (2001) emphasize that the agents they construct are dynamically consistent. The axiom system of Artzner et al. (1999) has inspired a number of papers on multiperiod risk measures, including Artzner et al. (2003), Riedel (2003), Weber (2003), and Frittelli and Rosazza Gianin (2004). In each of these contributions an axiom of dynamic consistency is 
imposed, although the precise content given to the axiom differs between papers. Cochrane and Saá Requejo (2000), in their discussion of "good-deal bounds" in a multiperiod setting, assume that the uncertainty structures in successive periods are independent of each other, which is analogous to the product property that we have discussed here.

Epstein and Schneider (2003) adopt an axiom of dynamic consistency as well, but they also add a discussion of the applicability of the axiom. As it is the case with all axioms concerning decision making, it is indeed important to be aware of situations where seemingly natural rules may be violated. Epstein and Schneider provide an example of a situation in which a rational agent might well show time-inconsistent behavior. A key role in this example is played by the fact that ambiguity may be increased, rather than decreased, by the arrival of new information. Ambiguity aversion may lead an agent to avoid such situations, and this may lead to time-inconsistencies. It has in fact already been demonstrated in the classical work by Kahneman and Tversky (1979) that human decisions tend to be sensitive to the order in which choices are presented.

While it is difficult to summarize human decision making in a set of rules, a more normative approach may be appropriate for decision making in financial institutions, or for purposes of regulation. In particular it seems reasonable to exclude situations as in Example 2.4, where a particular product is considered acceptable at price 48 whereas in all scenarios the accepted price at the next time instant would be only 40 . The axiom of dynamic consistency does indeed prevent such situations from occurring.

The property of absence of strictly acceptable opportunities is a strengthening of the property of absence of arbitrage. Although of course in actual markets it may happen that strictly acceptable opportunities exist, just as arbitrage opportunities may sometimes arise, we believe that the NSAO property is useful as a starting point for analysis. We have shown above that the NSAO property is implied by dynamic consistency. It should be noted though that the implication only goes one way, and that absence of strictly acceptable opportunities may well hold in situations where the axiom of dynamic consistency as formulated above is not satisfied, as illustrated by Example 3.1. Therefore, taking the "product closure" of a given collection of test measures can be a rather conservative way of ensuring that NSAO is satisfied. At this moment we are not aware however of any other systematic way of extending a given collection of test measures that achieves compliance with NSAO in a less conservative manner. Forming the product-type completion has the additional benefit of removing the type of anomaly found in Example 2.4, which is not always the case with alternative methods of achieving NSAO (cf. Remark 3.3).

Typical examples of collections of test measures that do not satisfy the product property, and that therefore do not represent dynamically consistent acceptability measures in the sense of this paper, are collections of measures formed on the basis of unknown-but-constant 
volatility. Such collections are formed by allowing a range of possible volatilities at each time step, but not allowing the volatility to vary from one time step to the other. In this way one obtains a collection of measures parametrized by a single volatility parameter. Models of this type assume that there is a true constant volatility, but we are just uncertain as to the exact value of this parameter. One may argue that, if such a supposition would be true, observations from the past would already have enabled us to estimate the actual volatility parameter to a high accuracy.

The product property expresses that the uncertainty about the whole multi-period probability measure takes the form of uncertainty about each single-period conditional probability measure separately. A possible source of the latter uncertainty could simply be the changeability of the world in which we live. It is important to note that the product property does allow this uncertainty to be state-dependent. The choice of suitable state variables provides a way to introduce model assumptions concerning the uncertainty introduced at each step in different situations.

Example 4.1 Consider the following stylized example with $N$ periods and events $A=$ $\left\{\alpha_{+}, \alpha_{0}, \alpha_{-}\right\}$corresponding to relative price changes given by $1+\varepsilon, 1$, and $1-\varepsilon$, where $0<\varepsilon<1$. Let $\mathbb{P}_{q}$ denote the one-step measure assigning probabilities $(1-q) / 2, q$, and $(1-q) / 2$ to the events $\alpha_{+}, \alpha_{0}$, and $\alpha_{-}$, respectively. Uncertain volatility can be modelled by considering test sets of the form $\mathcal{P}=\left\{\mathbb{P} \mid \mathbb{P}^{\mathrm{s}}\left(\cdot \mid \omega^{\prime}\right)=\mathbb{P}_{q}\right.$ for some $\left.q \in I\right\}$, for a given subinterval $I=\left[q_{\text {low }}, q_{\text {high }}\right]$ of $[0,1]$. The modeling can be refined by making the interval $I$ history-dependent. For example, the interval might depend on an exponentially weighted average of the occurrence of event $\alpha_{0}$ in the string $\omega^{\prime}$. Many alternatives are possible, at the cost of extending the state variable; for instance $I\left(\omega^{\prime}\right)$ could be made to depend on implied volatilities that are observed in the market. In a similar way one could consider multinomial distributions obtained from discretizing other popular models in finance, such as sets of lognormal distributions with mean and variance ranging over state-dependent intervals in each period.

\section{Conclusions}

We have considered the multiperiod extension of the notion of coherent risk measures as introduced by Artzner et al. (1999). Our main conclusions are that the addition of a dynamic consistency axiom leads to a simple and attractive characterization of coherent dynamic acceptability measures, and that the framework obtained in this way allows characterizations of price bounds for derivative assets in incomplete markets that are natural counterparts of the well-known results for the complete market case. 
We have worked within the simplest possible setting that allows consideration of partially revealed information and dynamic hedging. Interpreting our results from a continuous-time perspective, one might say that the product property would favor approaches that measure discrepancy between models in terms of an entropy rate, such as Pathak (2002), Trojani and Vanini (2002), and Sbuelz and Trojani (2002), above approaches that work with total relative entropy as a distance measure. ${ }^{9}$ In the absence of axiomatic underpinnings of continuous-time utility specifications, however, it remains difficult to make any definitive statements. Steps towards axiomatization of the coherent framework in a continuous-time setting have been taken by Delbaen (2003).

In this paper we have worked with the axiom of positive homogeneity, following Artzner et al. (1999). In some applications, such as portfolio optimization, this axiom may not be attractive. To accommodate these situations, the framework of Artzner et al. (1999) can be extended by adding a function that associates to each test measure a corresponding "floor"; cf. Carr et al. (2001), Föllmer and Schied (2002a-c). It has been shown that this extension corresponds to replacing the homogeneity axiom of Artzner et al. (1999) by a convexity axiom, and that, under suitable continuity assumptions, the representation (1.2) of Gilboa and Schmeidler (1989) can be obtained from the thus modified axiom scheme, even though this scheme is different from the one used by Gilboa and Schmeidler (Föllmer and Schied (2002a, Thm. 5, Cor. 14)). Naturally it would be of interest to extend these results to the intertemporal setting; this may lead to alternative axiomatic foundations for the dynamic Gilboa-Schmeidler specifications of Wang (2003) and Epstein and Schneider (2003).

For applications to pricing in incomplete markets, a key challenge is to find robust utility specifications that are (i) tractable, (ii) stable in the sense that calibrated parameters do not vary erratically over time, and (iii) successful in the sense that price bounds for exotic derivatives are tight and in conformity with market practice. The development of this paper suggests certain forms of such specifications and certain properties that may be considered attractive. However, this is of course still a long way from providing concrete specifications that are suitable for particular markets, and much exploratory work in this direction remains to be done.

\section{Appendix}

\section{Proof of Thm. 3.2}

We need the following definition.

\footnotetext{
${ }^{9}$ See for instance Mäkilä et al. (1999) and the references therein for analogous developments in control theory.
} 
Definition 6.1 A set of vectors $\left\{x_{1}, \ldots, x_{k}\right\} \subset \mathbb{R}^{n}$ is said to be positively complete if there exists no vector $g \in \mathbb{R}^{n}$ such that $g^{\top} x_{i} \geq 0$ for all $i$ and $g^{\top} x_{i}>0$ for some $i$.

An equivalent formulation is that the nonnegative cone generated by the vectors $x_{i}$ is the same as the linear span of these vectors; this motivates our terminology. If for each $i=$ $1, \ldots, N$ we have a set $X_{i}$ of vectors in $\mathbb{R}^{n_{i}}$, we can form the product set $X=X_{1} \times \cdots \times X_{N}$ which is defined by

$$
X:=\left\{\left[\begin{array}{c}
x_{1} \\
\vdots \\
x_{N}
\end{array}\right] \mid x_{1} \in X_{1}, \ldots, x_{N} \in X_{N}\right\} \subset \mathbb{R}^{n}
$$

where $n=n_{1}+\cdots+n_{N}$. For instance, the product of a set of three vectors in $\mathbb{R}^{2}$ and a set of four vectors in $\mathbb{R}^{3}$ is a set of twelve vectors in $\mathbb{R}^{5}$. We can now formulate the following lemma.

LEMma 6.2 A product of positively complete sets is again positively complete.

Proof For $i=1, \ldots, N$, let $X_{i}$ denote a set of $k_{i}$ vectors in $\mathbb{R}^{n_{i}}$, and suppose that each set $X_{i}$ is positively complete. Suppose $\left(g_{1}, \ldots, g_{N}\right) \in \mathbb{R}^{n_{1}} \times \cdots \times \mathbb{R}^{n_{N}}$ is such that

$$
\sum_{i=1}^{N} g_{i}^{\top} x_{i} \geq 0
$$

for all $\left(x_{1}, \ldots, x_{N}\right) \in X_{1} \times \cdots \times X_{N}$. We have to show that these conditions imply that

$$
\sum_{i=1}^{N} g_{i}^{\top} x_{i}=0
$$

for all $\left(x_{1}, \ldots, x_{N}\right) \in X_{1} \times \cdots \times X_{N}$. Take a fixed sequence $\left(x_{2}, \ldots, x_{N}\right)$ in $X_{2} \times \cdots X_{N}$, and let $X_{1}$ consist of the vectors $x_{1}^{1}, \ldots, x_{k_{1}}^{1}$. By Stiemke's lemma (see for instance Mangasarian (1969), p. 32), there exist positive numbers $\alpha_{1}, \ldots, \alpha_{k_{1}}$ such that $\alpha_{1} x_{1}^{1}+\cdots+\alpha_{k_{1}} x_{k_{1}}^{1}=0$. It follows that

$$
0 \leq \sum_{j=1}^{k_{1}} \alpha_{j}\left(g_{1}^{\top} x_{j}^{1}+\sum_{i=2}^{N} g_{i}^{\top} x_{i}\right)=\sum_{j=1}^{k_{1}} \alpha_{j} \cdot \sum_{i=2}^{N} g_{i}^{\top} x_{i}
$$

so that in particular

$$
\sum_{i=2}^{N} g_{i}^{\top} x_{i} \geq 0
$$

Since $x_{2}, \ldots, x_{N}$ were chosen arbitrarily, it follows that the above relation holds for all collections $\left(x_{2}, \ldots, x_{N}\right)$. The reasoning can be repeated to show that $\sum_{i=3}^{N} g_{i}^{\top} x_{i} \geq 0$ for all collections $\left(x_{3}, \ldots, x_{N}\right)$. Continuing in this way, we finally find $g_{N}^{\top} x_{N} \geq 0$ for all $x_{N} \in X_{N}$. By the assumption in the lemma, it follows that actually we must have $g_{N}^{\top} x_{N}=0$ for all $x_{N}$. Repeating the same exercise for a different order of the indices, we find for all $i$ that $g_{i}^{\top} x_{i}=0$ for all $x_{i} \in X_{i}$. The claim of the lemma follows. 
Proof (of Thm. 3.2) Suppose that the condition of the theorem holds and let $g$ be a trading strategy such that $E_{\mathbb{P}} H^{g} \geq 0$ for all $\mathbb{P} \in \mathcal{P}$; we then have to show that in fact $E_{\mathbb{P}} H^{g}=0$ for all $\mathbb{P} \in \mathcal{P}$. To see this, note that we may write

$$
\begin{aligned}
E_{\mathbb{P}} H^{g} & =\sum_{\omega \in \Omega} \mathbb{P}(\omega)\left[\sum_{t=0}^{T-1} g_{t}^{\top}(\omega)\left(S_{t+1}(\omega)-S_{t}(\omega)\right)\right] \\
& =\sum_{\omega \in \Omega} \mathbb{P}(\omega)\left[\sum_{\omega^{\prime} \alpha \preceq \omega} g^{\top}\left(\omega^{\prime}\right)\left(S\left(\omega^{\prime} \alpha\right)-S\left(\omega^{\prime}\right)\right)\right] \\
& =\sum_{\omega^{\prime} \in \Omega^{\prime \prime}} g^{\top}\left(\omega^{\prime}\right) \sum_{\alpha \in A} \mathbb{P}\left(\omega^{\prime} \alpha\right)\left[S\left(\omega^{\prime} \alpha\right)-S\left(\omega^{\prime}\right)\right] .
\end{aligned}
$$

Lemma 6.2 applies with the collections of vectors $\left\{\sum_{\alpha \in A} \mathbb{P}\left(\omega^{\prime} \alpha\right)\left[S\left(\omega^{\prime} \alpha\right)-S\left(\omega^{\prime}\right)\right] \mid \mathbb{P} \in \mathcal{P}\right\}$ playing the role of the collections $X_{i}$, and the set of partial trajectories $\Omega^{\prime \prime}$ playing the role of the index set $\{1, \ldots, N\}$. The statement in the theorem follows in this way from the assumption on the single-period economies.

\section{Proof of Thm. 3.4}

Take a product $X$ and a partial history $\omega^{\prime}$; let $\tau=\tau\left(\omega^{\prime}\right)$. To show that the left hand side of $(3.8)$ is at most equal to the right hand side, take a strategy $g \in \mathbb{G}$. We have:

$$
\begin{aligned}
\phi^{g}\left(X \mid \omega^{\prime}\right) & =\phi\left(X+H_{\tau}^{g} \mid \omega^{\prime}\right) \\
& =\inf _{P \in \mathcal{P}^{\mathrm{s}}\left(\omega^{\prime}\right)} E_{P} \phi\left(X+H_{\tau}^{g} \mid \omega^{\prime} \alpha\right) \\
& =\inf _{P \in \mathcal{P}^{\mathrm{s}}\left(\omega^{\prime}\right)} E_{P} \phi\left(X+H_{\tau+1}^{g}+g\left(\omega^{\prime}\right)^{\top}\left(S\left(\omega^{\prime} \alpha\right)-S\left(\omega^{\prime}\right)\right) \mid \omega^{\prime} \alpha\right) \\
& =\inf _{P \in \mathcal{P}^{\mathrm{s}}\left(\omega^{\prime}\right)} E_{P}\left[\phi\left(X+H_{\tau+1}^{g} \mid \omega^{\prime} \alpha\right)+g\left(\omega^{\prime}\right)^{\top}\left(S\left(\omega^{\prime} \alpha\right)-S\left(\omega^{\prime}\right)\right)\right] \\
& \leq \inf _{P \in \mathcal{P}^{\mathrm{s}}\left(\omega^{\prime}\right)} E_{P}\left[\phi^{*}\left(X \mid \omega^{\prime} \alpha\right)+g\left(\omega^{\prime}\right)^{\top}\left(S\left(\omega^{\prime} \alpha\right)-S\left(\omega^{\prime}\right)\right)\right] \\
& \leq \sup _{\gamma \in G} \inf _{P \in \mathcal{P}^{\mathrm{s}}\left(\omega^{\prime}\right)} E_{P}\left[\phi^{*}\left(X \mid \omega^{\prime} \alpha\right)+\gamma^{\top}\left(S\left(\omega^{\prime} \alpha\right)-S\left(\omega^{\prime}\right)\right)\right] .
\end{aligned}
$$

Since this holds for every $g \in \mathbb{G}$, we find

$$
\phi^{g}\left(X \mid \omega^{\prime}\right) \leq \sup _{\gamma \in G} \inf _{P \in \mathcal{P}^{\mathrm{s}}\left(\omega^{\prime}\right)} E_{P}\left[\phi^{*}\left(X \mid \omega^{\prime} \alpha\right)+\gamma^{\top}\left(S\left(\omega^{\prime} \alpha\right)-S\left(\omega^{\prime}\right)\right)\right]
$$

which completes the first part of the proof. To show the reverse inequality, take $\gamma \in G$ and $\varepsilon>0$, and let $g$ be a strategy such that

$$
\phi^{g}\left(X \mid \omega^{\prime} \alpha\right) \geq \phi^{*}\left(X \mid \omega^{\prime} \alpha\right)-\varepsilon
$$


and $g\left(\omega^{\prime}\right)=\gamma$. We have

$$
\begin{aligned}
\phi^{*}\left(X \mid \omega^{\prime}\right) & \geq \phi^{g}\left(X \mid \omega^{\prime}\right) \\
& =\inf _{P \in \mathcal{P}^{\mathrm{S}}\left(\omega^{\prime}\right)} E_{P}\left[\phi^{g}\left(X \mid \omega^{\prime} \alpha\right)+\gamma^{\top}\left(S\left(\omega^{\prime} \alpha\right)-S\left(\omega^{\prime}\right)\right)\right] \\
& \geq \inf _{P \in \mathcal{P}^{\mathrm{S}}\left(\omega^{\prime}\right)} E_{P}\left[\phi^{*}\left(X \mid \omega^{\prime} \alpha\right)+\gamma^{\top}\left(S\left(\omega^{\prime} \alpha\right)-S\left(\omega^{\prime}\right)\right)\right]-\varepsilon .
\end{aligned}
$$

Since this holds for every $\gamma \in G$ and every $\varepsilon>0$, we can take supremum with respect to both $\gamma$ and $\varepsilon$ and obtain

$$
\phi^{*}\left(X \mid \omega^{\prime}\right) \geq \sup _{\gamma \in G} \inf _{P \in \mathcal{P}^{\mathrm{s}}\left(\omega^{\prime}\right)} E_{P}\left[\phi^{*}\left(X \mid \omega^{\prime} \alpha\right)+\gamma^{\top}\left(S\left(\omega^{\prime} \alpha\right)-S\left(\omega^{\prime}\right)\right)\right]
$$

as required.

Concerning the second statement, take a strategy $g \in \mathbb{G}$ and a product $X: \Omega \rightarrow \mathbb{R}$. The proof proceeds by induction with respect to the difference of final time and current time. For complete histories $\omega \in \Omega$, the normalization property implies that $\phi^{g}(X \mid \omega)=X(\omega)$ for all strategies $g$ and so the condition (3.9) is trivially satisfied. Now assume that (3.9) holds for all partial histories of length $\tau+1$, and let $\omega^{\prime}$ be a sequence of length $\tau$. Then we can write:

$$
\begin{aligned}
\phi^{g}\left(X \mid \omega^{\prime}\right) & =\phi\left(X+H_{\tau}^{g} \mid \omega^{\prime}\right) \\
& =\inf _{P \in \mathcal{P}^{\mathbf{s}}\left(\omega^{\prime}\right)} E_{P} \phi\left(X+H_{\tau}^{g} \mid \omega^{\prime} \alpha\right) \\
& =\inf _{P \in \mathcal{P}^{\mathbf{s}}\left(\omega^{\prime}\right)} E_{P}\left[\phi\left(X+H_{\tau+1}^{g} \mid \omega^{\prime} \alpha\right)+g\left(\omega^{\prime}\right)^{\top}\left(S\left(\omega^{\prime} \alpha\right)-S\left(\omega^{\prime}\right)\right)\right] \\
& \leq \inf _{P \in \mathcal{P}^{\mathbf{s}}\left(\omega^{\prime}\right)} E_{P}\left[\phi^{*}\left(X \mid \omega^{\prime} \alpha\right)+g\left(\omega^{\prime}\right)^{\top}\left(S\left(\omega^{\prime} \alpha\right)-S\left(\omega^{\prime}\right)\right)\right] \\
& \leq \sup _{\gamma \in \mathbb{G}} \inf _{P \in \mathcal{P}^{\mathbf{s}}\left(\omega^{\prime}\right)} E_{P}\left[\phi^{*}\left(X \mid \omega^{\prime} \alpha\right)+\gamma^{\top}\left(S\left(\omega^{\prime} \alpha\right)-S\left(\omega^{\prime}\right)\right)\right] \\
& =\inf _{P \in \mathcal{P}^{\mathbf{s}}\left(\omega^{\prime}\right)} E_{P}\left[\phi^{g^{*}}\left(X \mid \omega^{\prime} \alpha\right)+\left(g^{*}\left(\omega^{\prime}\right)\right)^{\top}\left(S\left(\omega^{\prime} \alpha\right)-S\left(\omega^{\prime}\right)\right)\right] \\
& =\inf _{P \in \mathcal{P}^{\mathbf{s}}\left(\omega^{\prime}\right)} E_{P} \phi\left(X+H_{\tau}^{g^{*}} \mid \omega^{\prime} \alpha\right) \\
& =\phi^{g^{*}}\left(X \mid \omega^{\prime}\right) .
\end{aligned}
$$

This completes the induction step. The statement of the theorem follows.

\section{Proof of Prop. 3.5}

We show how this proposition can be seen as a special case of Prop. 20 in Föllmer and Schied (2002a). Acceptability measures can be represented by penalty functions, as follows. Let $L^{0}(\Omega)$ denote the space of probability measures on $\Omega$. The penalty function $\alpha: L^{0}(\Omega) \rightarrow \mathbb{R} \cup$ $\{\infty\}$, defined by $\alpha(\mathbb{P})=0$ if $\mathbb{P} \in \mathcal{P}$, else $\alpha(\mathbb{P})=\infty$, represents $\phi_{\mathcal{P}}=\inf _{P \in L^{0}(\Omega)} E_{\mathbb{P}}[\cdot]+\alpha(P)$. The minimal penalty function $\alpha_{0}$ is the penalty function of the closed convex hull of $\mathcal{P}$; this induces the same acceptability measure as $\mathcal{P}$ itself does (cf. Prop. 7 in the cited paper). 
Applying Prop. 20 (all conditions are satisfied) with $\alpha_{0}^{0}$ equal to the penalty function of the closed convex hull of $\mathcal{P}$ now yields that $\phi^{*}=\left(\phi_{\mathcal{P}}\right)^{*}=\inf _{\mathbb{P} \in L^{0}(\Omega)} E_{\mathbb{P}}[\cdot]+\alpha(\mathbb{P})+\alpha^{\mathbb{G}}(\mathbb{P})$ with $\alpha^{\mathbb{G}}: L^{0}(\Omega) \rightarrow \mathbb{R} \cup\{\infty\}$ defined by $\alpha^{\mathbb{G}}(\mathbb{P}):=\sup _{g \in \mathbb{G}} E_{\mathbb{P}}\left[H^{g}\right]$, cf. also Prop. 4.50 in Föllmer and Schied (2002c). For a linear hedge set this amounts to $\alpha^{\mathbb{G}}(\mathbb{P})=0$ if $\mathbb{P} \in \mathcal{P}_{0}$, and otherwise $\alpha^{\mathbb{G}}(\mathbb{P})=\infty$. The result follows.

\section{References}

Anscombe, F., and R. Aumann, 1963. Definition of subjective probability. Annals of Mathematical Statistics, 34, 199-205.

Artzner, Ph., F. Delbaen, J.-M. Eber, and D. Heath, 1999. Coherent measures of risk. Mathematical Finance, 9, 203-228.

Artzner, Ph., F. Delbaen, J.-M. Eber, D. Heath, and H. Ku, 2003. Coherent multiperiod risk adjusted values and Bellman's principle. Manuscript, Université Louis Pasteur.

Barberis, N., M. Huang, and T. Santos, 2001. Prospect theory and asset prices. Quarterly Journal of Economics, 116, 1-53.

Bernardo, A., and O. Ledoit, 2000. Gain-loss and asset pricing. Journal of Political Economy, 108, 144-172.

Carr, P., H. Geman, and D. B. Madan, 2001. Pricing and hedging in incomplete markets. Journal of Financial Economics, 32, 131-167.

Casadesus-Masanell, R., P. Klibanoff, and E. Ozdenoren, 2000. Maxmin expected utility over Savage acts with a set of priors. Journal of Economic Theory, 92, 35-65.

Chen, Z., and L.G. Epstein, 2002. Ambiguity, risk and asset returns in continuous time. Econometrica, 70, 1403-1443.

Černý, A., and S. Hodges, 2002. The theory of good-deal pricing in financial markets. In: H. Geman, D. Madan, S.R. Pliska, T. Vorst (eds.), Mathematical Finance - Bachelier Congress 2000, Springer, Berlin, pp. 175-202.

Cochrane, J., and J. Saá Requejo, 2000. Beyond arbitrage: good-deal asset price bounds in incomplete markets. Journal of Political Economy, 108, 79-119.

Cvitanić, J., and I. Karatzas, 1999. On dynamic measures of risk. Finance and Stochastics, 3, 451-482.

Delbaen, F., 2002. Coherent risk measures on general probability spaces. In: K. Sandmann, P. J. Schönbucher (eds.), Advances in Finance and Stochastics. Essays in Honour of Dieter Sondermann, Springer, Berlin, pp. 1-38. 
Delbaen, F., 2003. The structure of $m$-stable sets and in particular of the set of risk neutral measures. Manuscript, ETH Zürich.

Duffie, D., and L.G. Epstein, 1992. Stochastic differential utility. Econometrica, 60, 353394.

Ellsberg, D., 1961. Risk, ambiguity, and the Savage axioms. Quarterly Journal of Economics, 75, 643-669.

Epstein, L.G., and M. Schneider, 2003. Recursive multiple-priors. Journal of Economic Theory, 113, 1-31.

Epstein, L.G., and T. Wang, 1994. Intertemporal asset pricing under Knightian uncertainty. Econometrica, 62, 283-322.

Föllmer, H., and A. Schied, 2002a. Convex measures of risk and trading constraints. Finance and Stochastics, 6, pp. 429-447.

Föllmer, H., and A. Schied, 2002b. Robust preferences and convex measures of risk. In: K. Sandmann, P. J. Schönbucher (eds.), Advances in Finance and Stochastics. Essays in Honour of Dieter Sondermann, Springer, Berlin, pp. 39-56.

Föllmer, H., and A. Schied, 2002c. Stochastic Finance. An introduction in discrete time. De Gruyter Studies in Mathematics, Vol. 27, de Gruyter, Berlin / New York.

Frittelli, M. and M. Rosazza Gianin, 2004. Dynamic convex risk measures. In: G. Szegö (ed.), Risk Measures for the 21st Century, Wiley, New York.

Frittelli, M. and G. Scandolo, 2004. Risk measures and capital requirements for processes. Manuscript, Università degli Studi di Firenze.

Gilboa, I., and D. Schmeidler, 1989. Maxmin expected utility with non-unique prior. Journal of Mathematical Economics, 18, 141-153.

Hansen, L., and T. Sargent, 2001. Robust control and model uncertainty. American Economic Review, 91, 60-66.

Hansen, L., T. Sargent, G. Turmuhambetova, and N. Williams, 2001. Robustness and uncertainty aversion. Manuscript, University of Chicago.

Heston, S., 1993. A closed-form solution for options with stochastic volatility with applications to bond and currency options. Review of Financial Studies, 6, 327-343.

Huber, P., 1981. Robust Statistics. Wiley, New York.

Hull, J., and A. White, 1990. Pricing interest rate derivatives securities. Review of Financial Studies, 3, 573-592. 
Jaschke, S., and U. Küchler, 2001. Coherent risk measures and good-deal bounds. Finance and Stochastics, 5, 181-200.

Kahneman, D., and A. Tversky, 1979. Prospect theory: an analysis of decision under risk. Econometrica, 47, 263-291.

Kirch, M., 2003. Efficient Hedging in Incomplete Markets under Model Uncertainty, PhD thesis, Humboldt University, Berlin.

Madan, D., P. Carr, and E. Chang, 1998. The variance gamma process and option pricing. European Finance Review, 2, 79-105.

Maenhout, P., 2004. Robust portfolio rules and asset pricing. Forthcoming in Review of Financial Studies.

Mäkilä, P.M., J.R. Partington, and T. Norlander, 1999. Bounded power signal spaces for robust control and modelling. SIAM Journal on Control and Optimization, 37, 92-117.

Mangasarian, O. L., 1969. Nonlinear Programming. McGraw-Hill, New York.

Pathak, P.A., 2002. Notes on robust portfolio choice. Manuscript, Harvard University.

Riedel, F., 2003. Dynamic coherent risk measures. Manuscript, Stanford University.

Savage, L., 1954. Foundations of Statistics. Wiley, New York.

Sbuelz, A., and F. Trojani, 2002. Equilibrium asset pricing with time varying pessimism. CentER Discussion Paper 2002-102, Tilburg University.

Skiadas, C., 2003. Robust control and recursive utility. Finance and Stochastics, 7, 475-489. Trojani, F., and P. Vanini, 2002. A note on robustness in Merton's model of intertemporal consumption and portfolio choice. Journal of Economic Dynamics and Control, 26, 423-435.

Uppal, R., and T. Wang, 2003. Model uncertainty and under diversification. Journal of Finance, 58, 2465-2486.

Wang, T., 2003. Conditional preferences and updating. Journal of Economic Theory, 108, $286-321$.

Weber, S., 2003. Distribution-invariant dynamic risk measures. Manuscript, Humboldt University, Berlin. 